\title{
Facturation correcte de l'hématologie au laboratoire de cabinet médical: la FMH conteste la procédure de I'OFSP
}

Il y a quelques semaines, la FMH a reçu une première demande d'un médecin concernant la facturation des positions hématologiques. Il avait été invité à se prononcer par une caisse-maladie sur l'interprétation tarifaire adoptée par elle à la suite d'une lettre de l'OFAS (aujourd'hui OFSP); il justifia sa facturation en étant convaincu d'avoir toujours agi correctement. Peu de temps après, la caisse lui envoya une seconde lettre l'enjoignant de lui rembourser Fr. 10 000.- selon une proposition de règlement ad hoc.

L'attitude de la caisse-maladie provoqua une forte réaction au service juridique de la $\mathrm{FMH}$, non seulement parce qu'elle interprétait le droit de façon singulière mais avant tout parce qu'elle faisait montre d'une grande suffisance, au mépris de la procédure habituelle.

Il s'avéra bientôt qu'il ne s'agissait pas d'un cas unique mais d'une campagne générale de demandes de remboursement, basée sur une modification de la liste des analyses, encore au stade de projet. Le service juridique de l'OFSP confirma que cette modification n'avait pas été formellement approuvée par le chef du département ni même publiée, mais qu'elle émanait toutefois d'une circulaire à caractère obligatoire.

Nous ne pouvons pas accepter une telle procédure «sauvage». Le droit ne peut être modifié et communiqué que dans le cadre prévu par la loi: on respecte ainsi les citoyens en leur donnant les moyens d'agir en conséquence.
Cette situation et l'ampleur de cette campagne ont incité la FMH à intervenir auprès de l'OFSP. Dans sa lettre du 16 juillet 2004, elle défend la position suivante:

1. La facturation cumulée de positions individuelles dans les cas d'analyses automatisées n'est pas correcte déjà d'après le droit actuel, et les caisses-maladie peuvent la contester.

2. A l'inverse, cette facturation est correcte lorsque les déterminations sont effectuées manuellement dans le laboratoire du cabinet médical.

Le cumul maximal de déterminations manuelles (positions 8210.00, 8273.00, 8275.00, 8406.00 et 8560.00 ), comme prévu dans la lettre de l'OFSP (page 4, chiffre 2), ne ressort pas du texte juridiquement valable.

De plus, la FMH souligne que des dispositions juridiques ne peuvent être modifiées que par l'organe disposant de la compétence décisionnelle et selon la procédure prévue à cet effet. La FMH demande à l'OFSP d'inviter les caisses-maladie à accepter les factures établies selon le droit actuel.

Les lecteurs intéressés trouveront la lettre de la FMH à l'OFSP sur notre site internet www. fmh.ch $\rightarrow$ français $\rightarrow$ Nos prestations $\rightarrow$ Tarifs $\rightarrow$ Autres tarifs $\rightarrow$ BSV 